\title{
ECMPride: Prediction of human extracellular matrix proteins based on the ideal dataset using hybrid features with domain evidence
}

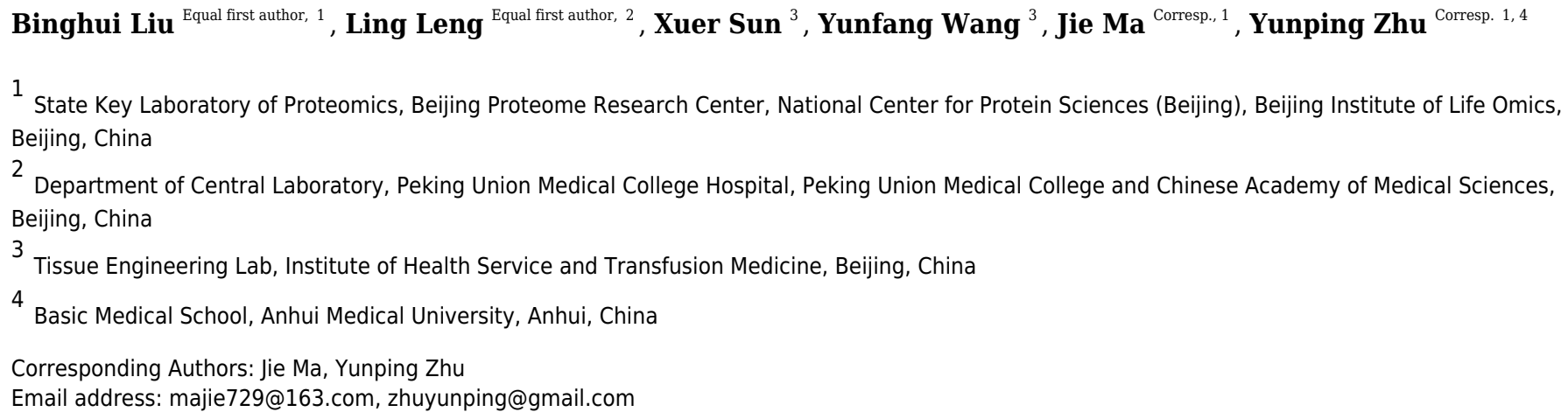

Extracellular matrix (ECM) proteins play an essential role in various biological processes in multicellular organisms, and their abnormal regulation can lead to many diseases. For large-scale ECM protein identification, especially through proteomic-based techniques, a theoretical reference database of ECM proteins is required. In this study, based on the experimentally verified ECM datasets and by the integration of protein domain features and a machine learning model, we developed ECMPride, a flexible and scalable tool for predicting ECM proteins. ECMPride achieved excellent performance in predicting ECM proteins, with appropriate balanced accuracy and sensitivity, and the performance of ECMPride was shown to be superior to the previously developed tool. A new theoretical dataset of human ECM components was also established by applying ECMPride to all human entries in the SwissProt database, containing a significant number of putative ECM proteins as well as the abundant biological annotations. This dataset might serve as a valuable reference resource for ECM protein identification. 
1 ECMPride: Prediction of human extracellular matrix

2 proteins based on the ideal dataset using hybrid

3 features with domain evidence

6 Binghui Liu ${ }^{1}$, Ling Leng ${ }^{2}$, Xuer Sun ${ }^{3}$, Yunfang Wang ${ }^{3}$, Jie Ma ${ }^{1}$, Yunping Zhu ${ }^{1,4}$

$8 \quad{ }^{1}$ State Key Laboratory of Proteomics, Beijing Proteome Research Center, National Center for

9 Protein Sciences (Beijing), Beijing Institute of Life Omics, Beijing, China.

$10{ }^{2}$ Department of Central Laboratory, Peking Union Medical College Hospital, Peking Union

11 Medical College and Chinese Academy of Medical Sciences, Beijing, China.

$12{ }^{3}$ Tissue Engineering Lab, Institute of Health Service and Transfusion Medicine, Beijing, China.

$13{ }^{4}$ Basic Medical School, Anhui Medical University, Anhui, China.

14

Corresponding Authors:

Jie Ma

17 State Key Laboratory of Proteomics, Beijing Proteome Research Center, National Center for

18 Protein Sciences (Beijing), Beijing Institute of Life Omics, Beijing, 102206, China.

19 Email address: majie729@163.com

20 Yunping Zhu

21 State Key Laboratory of Proteomics, Beijing Proteome Research Center, National Center for

22 Protein Sciences (Beijing), Beijing Institute of Life Omics, Beijing, 102206, China.

23 Email address: zhuyunping@gmail.com 


\section{Abstract}

26 Extracellular matrix (ECM) proteins play an essential role in various biological processes in

27

28

29

30

31

32

33

34

35

36

37

38

39

40

41

42

43

44

45

46

47

48

49

50

51

52

53

54

55

56

57

58

59

60

61

62

63

64

multicellular organisms, and their abnormal regulation can lead to many diseases. For large-scale ECM protein identification, especially through proteomic-based techniques, a theoretical reference database of ECM proteins is required. In this study, based on the experimentally verified ECM datasets and by the integration of protein domain features and a machine learning model, we developed ECMPride, a flexible and scalable tool for predicting ECM proteins. ECMPride achieved excellent performance in predicting ECM proteins, with appropriate balanced accuracy and sensitivity, and the performance of ECMPride was shown to be superior to the previously developed tool. A new theoretical dataset of human ECM components was also established by applying ECMPride to all human entries in the SwissProt database, containing a significant number of putative ECM proteins as well as the abundant biological annotations. This dataset might serve as a valuable reference resource for ECM protein identification.

\section{Introduction}

The extracellular matrix $(\mathrm{ECM})$ is a vital component of the cellular microenvironment, providing structural and functional support to surrounding cells (Bonnans et al. 2014; Theocharis et al. 2016). ECM proteins play crucial roles in regulating diverse functions of cells, including differentiation, proliferation, survival, and migration (Bonnans et al. 2014; Hynes 2009), and their dysregulation can result in a wide range of diseases (Bateman et al. 2009; Liu et al. 2019; Tokhmafshan et al. 2017; Walker et al. 2018). A better understanding of the composition and function of ECM proteins should contribute to useful therapeutic targets for related diseases.

The rapid development of multi-omics research has substantially benefited ECM identification and characterization. However, for large-scale ECM protein identification, especially for proteomics-based techniques, a general reference database of ECM proteins is required. Many strategies have been developed by the researchers to define the set of ECM proteins, including the molecular fishing method (Cain et al. 2009), the systematic curation method (Cromar et al. 2012), and the domain-based method (Naba et al. 2016). Besides, Richard-Blum lab established the MatrixDB database, which is focused on the interactions established by extracellular proteins and polysaccharides and can provide interaction evidence for putative ECMs validation (Clerc et al. 2018). Domain architectures change during evolution (Apic et al. 2003), and proteins with the same domain architecture are frequently related (Bornberg-Bauer \& Alba 2013). By utilizing the domain-based structure of ECM proteins, Naba et al. used an in silico approach to define ECM components and, based on this, constructed the Matrisome database in 2012 (Naba et al. 2012). The Matrisome has become a general reference database for proteomics-based ECM research in recent years (Åhrman et al. 2018; Gopal et al. 2017; Lennon et al. 2014; Mayorca-Guiliani et al. 2017). Further, Naba et al. presented the first draft of the ECM atlas, which was established by integrating publicly available mass spectrometry data from studies explicitly designed to characterize the global composition of ECM proteins (Naba et al. 2016). However, when

Peer) reviewing PDF | (2019:12:43724:2:1:NEW 20 Mar 2020) 
65

66

67

68

69

70

71

72

73

74

75

76

77

78

79

80

81

82

83

84

85

86

87

88

89

90

91

92

93

94

95

96

97

98

99

100

101

102

103

104

compared with Matrisome, there is relatively low overlap $\sim 51 \%$ ( 73\% for Core matrisome and $\sim 42 \%$ for Matrisome-associated) between experimentally identified ECMs and theoretically predicted ones, which likely reflects the poor representation of insoluble matrix tissues in the experimental datasets used for comparison. Additionally, the in silico Matrisome was constructed via a semi-empirical and manual-assisted approach, so there are some difficulties for the database in dealing with the problems of constant updating and expansion to other species.

Several attempts have also been made by bioinformatics researchers to predict ECM proteins based on machine learning methods; specifically, a series of tools were developed, including ECMPP (Jung et al. 2010), EcmPred (Kandaswamy et al. 2013), PECM (Zhang et al. 2014), IECMP (Yang et al. 2015), ECMP-HybKNN (Ali \& Hayat 2016), BAMORF (Guan et al. 2017), and TargetECMP (Kabir et al. 2018). Most tools were developed based on a generic pipeline, which uses different machine learning algorithms to build classification models on the extracted features and training datasets and can achieve automated prediction of ECM proteins. The most significant shortcoming of these tools is their lack of a connection with experimental biological features, especially concerning standard dataset construction and classification feature extraction (Article S1). In addition, there are no tools available other than EcmPred.

In summary, the Matrisome database presented by Naba et al. compiles in silico and in vivo data on ECM proteins, and the existing bioinformatics prediction tools for ECMs are robust in modeling. Thus, in this study, we proposed incorporating these advantages of both approaches and developed ECMPride, a flexible and scalable tool for predicting extracellular matrix proteins. Based on the experimentally verified ECM datasets, while integrating protein domain features and a machine learning model, ECMPride achieved better performance when compared with EcmPred. We also provide researchers with a comprehensive dataset of all putative human ECMs (named ECMPrideDB) by applying ECMPride to all human protein sequences in the SwissProt database (Consortium 2017), and this ECM dataset might serve as a valuable reference resource for future investigations.

\section{Materials \& Methods}

\section{Datasets}

The standard training dataset consists of a positive dataset of ECM proteins and a negative dataset of non-ECM proteins (Table S1). The positive one consists of the 521 human proteins whose ECM-related status is supported by Matrisome with further credible evidence (Naba et al. 2016) (Table S2). In contrast, the negative one consists of 11336 human intracellular proteins from the Human Protein Atlas database developed by Thul et al. (Thul et al. 2017). The detailed process of generating positive and negative datasets, as well as the Matrisome categories of the positive dataset, can be found in Article S1.

\section{Feature Extraction}


105 Three main classes and 167 features in total are introduced into ECMPride to represent the 106 characteristics of ECM proteins, including ECM protein-related structural domains (from now on 107 referred to as ECM domains) (Naba et al. 2012), physicochemical properties (Kandaswamy et al. 108 2013), and position-specific scoring matrix (PSSM) (Altschul et al. 1997) (all features are listed 109 in Table S3).

110

111

\section{ECM domains}

We are the first to introduce domain into machine learning algorithms to predict ECM systematically. ECM proteins typically include multiple, independently folded domains whose sequences and arrangements are highly conserved (Hynes 2009). Based on this hallmark, Naba et al. established a list of "inclusion domains" commonly found in ECM proteins and a list of "exclusion domains" whose presence ruled a protein out from being a part of the ECM (Naba et al. 2012). These two lists are first merged, and then, domains that are not in the version of InterPro 69.0 (Mitchell et al. 2018) or do not exist in any protein of the dataset are excluded. Finally, a list of 63 ECM domains is obtained (Table S3).

The score for $i$-th ECM domain $\mathrm{D}_{i}$ of protein $A$ is represented as follows:

123

124

125

126

127

128

129

130

131

132

133

134

135

136

137

138

139

140

141

142

$$
X_{i}=\left\{\begin{array}{l}
0\left(\text { if } \mathrm{D}_{i} \in A\right) \\
1\left(\text { if } \mathrm{D}_{i} \notin A\right)
\end{array}(\mathrm{i}=1,2, \ldots 63)\right.
$$

Here, the evidence of whether $\mathrm{D}_{i}$ belongs to $A$ comes from SwissProt (Consortium 2017).

Finally, a 63-D feature vector of ECM domains is constructed for every protein sequence.

\section{Position-Specific Scoring Matrix (PSSM)}

For protein evolution, sequences evolve via the substitution, insertion, or deletion of residues (Chou \& Shen 2007). After a long time, the accumulation of these changes slowly eliminates the similarities between the original protein and the final protein; however, some of the critical residues associated with the essential properties of the protein remain stable, which is referred to as evolutionary conservation (Zhang et al. 2014). Such conservation usually occurs in sequences with important biological functions (Zuo et al. 2014). Therefore, evolutionary information is critical to the prediction of protein structure and function (Ding et al. 2014).

PSSM is a matrix that can well reflect the evolution information of a protein. It is generated by running PSI-BLAST (Altschul et al. 1997) in the database of SwissProt through three iterations, with 0.001 as an E-value cut-off. As shown below, it consists of $20 \times \mathrm{L}$ elements, with $\mathrm{L}$ representing the length of the protein sequence. 


$$
P_{P S S M}=\left[\begin{array}{cccccc}
E_{1,1} & E_{1,2} & \cdots & E_{1, j} & \cdots & E_{1,20} \\
E_{2,1} & E_{2,2} & \cdots & E_{2, j} & \cdots & E_{2,20} \\
\vdots & \vdots & \cdots & \vdots & \cdots & \vdots \\
E_{i, 1} & E_{i, 2} & \cdots & E_{i, j} & \cdots & E_{i, 20} \\
\vdots & \vdots & \cdots & \vdots & \cdots & \vdots \\
E_{L, 1} & E_{L, 2} & \cdots & E_{L, j} & \cdots & E_{L, 20}
\end{array}\right]
$$

144

145 Here, $E_{i, j}$ represents the score of the amino acid mutation in the $i$-th position of the sequence to 146 form the amino acid type $j$ during evolution. Then, PSSM is converted into an 80-D vector by 147 standardization and grey model theory (The detailed process of conversion could be found in 148 Article S1) (Chou 2001; Matsuda et al. 2005).

149

150

151

152

153

154

155

156

157

158

159

160

161

162

163

164

165

166

167

168

169

170

171

172

173

174

175

\section{Physicochemical Properties}

The structure and function of proteins are defined by the physicochemical properties of the 20 amino acids, which have been the subject of a large number of experimental and theoretical studies. The physicochemical properties of the 20 amino acids can be represented by a set of 20 values of an amino acid index (AAIndex) (Kawashima et al. 2007). There is now a database exclusively dedicated to storing AAIndex values (UMBC AAindex Database).

Here, we use 24 physicochemical properties selected by Kandaswamy et al. (Kandaswamy et al. 2013) from the UMBC AAindex Database (Table S4). The formula for calculating each physicochemical property of a protein is as follows:

(1)

(1)

Where AAIndex ${ }_{i}$ is the AAIndex value of the physicochemical property corresponding to the $i$ th amino acid in the protein sequence, and $\mathrm{L}$ is the length of the protein sequence. Finally, a $24-$ $\mathrm{D}$ feature vector of physicochemical properties is established for every protein sequence.

\section{Feature Selection}

For feature selection, we first perform feature importance scoring. This involves scoring the importance of all of the extracted features by the Maximum Relevance Minimum Redundancy (mRMR) algorithm (Peng et al. 2005) (The detailed process is shown in Article S1). The features are ranked according to the order of the scores from high to low.

Next, we adopt the Incremental Feature Selection (IFS) method to obtain the optimal feature subset based on the ranked feature set. The process begins with an empty feature set and adds features one by one in order of importance from high to low. Each time a feature is added, a new 
176 feature subset is generated so that $n$ features will generate $n$ feature subsets (Lin et al. 2013). The 177 subset of features with better predictive performance and fewer features would be considered the 178 optimal feature subset (Yang et al. 2015).

179

\section{Prediction model and Performance evaluation}

In this study, the Random forest model has been implemented in ECMPride for prediction. Developed by Breiman, the Random Forest algorithm is an integrated classifier consisting of numerous decision trees. It uses the bootstrap method to extract multiple identical samples from the original sample to generate a training set and then builds a decision tree with each sample in the training set. Finally, the final prediction result of the Random Forest model is obtained by voting on all decision tree prediction results (Breiman 2001). Random Forests have high predictive accuracy, have good tolerance of outliers and noise, and are not prone to over-fitting. They can handle both continuous and discrete variables, making them advantageous and increasingly mature machine learning algorithms. Here we use the randomForest package of $\mathrm{R}$ to implement the classification of ECM and non-ECM components (Liaw \& Wiener 2002).

Ten-fold cross-validation is used to evaluate the predictive model, and the under-sampling ensemble method is implemented to overcome the imbalance of the training datasets. Meanwhile, we employed the following four parameters to evaluate the performance of the ECM prediction models: sensitivity $(S n)$, specificity $(S p)$, accuracy $(A c c)$, and balanced accuracy $(B A c c)$. These can be represented by four indicators: true positive $(T P)$, false negative $(F N)$, true negative $(T N)$, and false positive $(F P)$. The detailed model training and parameters calculation can be found in Article S1.

\section{Results}

\section{Construction of ECMPride}

We built ECMPride, a command-line based tool that allows users to predict ECM proteins. The tool is available and freely downloaded from the public repository GitHub: https://github.com/Binghui-Liu/ECMPride.git. The overall workflow of ECMPride is shown in Fig. 1. As a high-quality ECM prediction tool, ECMPride has several unique features. First, positive and negative standard datasets (Table S1) are constructed based on reliable experimental and theoretical sources, including the Matrisome, ECM Atlas (Naba et al. 2016), the Human Protein Atlas (Thul et al. 2017) and Gene Ontology annotation (Consortium 2016), as well as a series of ECM proteomic studies (Table S2). Then, three main classes and 167 features in total are introduced into ECMPride to represent the characteristics of ECM proteins. In particular, the ECM domains proposed by Naba $e t$ al. are introduced into machine learning algorithms for the first time. In addition, the mRMR-IFS methods are implemented to reduce feature redundancy. Finally, to handle the classification problem of imbalanced datasets, the under-sampling ensemble method is employed for modeling (Table S5), and balanced accuracy is adopted as the 
215 essential criterion to evaluate the performance (Fig. S1). All details about the ECMPride pipeline

216 construction can be found in Materials \& Methods and Article S1.

217

218 ECMPride achieves good performance

219 ECMPride reduces feature redundancy to a certain extent via the feature selection step. All of the

220167 features are scored and sorted by mRMR (Table S3), and the IFS method is used to generate

221167 feature subsets and further generate 167 corresponding candidate models (Table S6). As

222 shown in Fig. 2, when the top 151 features are selected as the feature subset, the model achieves

223

224 the highest balanced accuracy of 0.9142, and the corresponding value is 0.9070 when all 167

225

226 features are used for prediction. Therefore, feature selection allows us to achieve better prediction with fewer features. In this context, ECMPride is established based on the top 151 features.

227

228

A series of tools had been developed by researchers to predict ECM proteins (Ali \& Hayat 2016;

229

230

231

232

233

234

235

236

237

238

239

240

241

242

243

244

245

246

247

248

249

250

251

252

253

254

Guan et al. 2017; Jung et al. 2010; Kabir et al. 2018; Kandaswamy et al. 2013; Yang et al. 2015; Zhang et al. 2014), so it's necessary to compare ECMPride with these tools. As the datasets used by ECMPride differ from the datasets used for previous tools, it is meaningless to compare their performance directly. Meanwhile, most of the previously released tools are no longer available for a variety of reasons, so it is impossible to compare ECMPride with such tools in an independent dataset. As such, we here attempt to reproduce the previous tools by carefully reviewing the articles about them; only for the tool EcmPred can localization be implemented well (Kandaswamy et al. 2013). Therefore, we applied ECMPride's and EcmPred's methods to each other's training dataset and compared their performance (Table 1). Using the same method (ECMPride or EcmPred), the model based on ECMPride's dataset (D1 in Table 1) behaved higher balanced accuracy than that based on EcmPred's dataset (D2 in Table 1), which means that the new training dataset is better than the old one. With the same dataset (D1 or D2 in Table 1), the model based on ECMPride's method behaved higher balanced accuracy than that based on EcmPred's method, which means that the method of ECMPride is better than that of EcmPred. Overall, ECMPride achieved better performance than EcmPred.

\section{Construction of theoretical reference dataset of human ECM proteins}

To obtained a comprehensive collection of theoretical human ECM proteins, we applied ECMPride to all human entries in the SwissProt database (Consortium 2017).

The proteins with a probability of being ECM higher than 0.7 are considered to be confidently predicted results. These proteins, together with the positive ECMs, are accepted as putative human ECM proteins and compose the theoretical reference dataset of human ECM proteins (named ECMPrideDB, Table S7). We also collected information on relevant databases to annotate genes in ECMPrideDB (Table S7), including Human Protein Atlas (Thul et al. 2017), ExoCarta (Keerthikumar et al. 2016) and GO (Consortium 2016). Then, we compared ECMPrideDB with Matrisome (Naba et al. 2016), as well as two experimental datasets generated

Peer) reviewing PDF | (2019:12:43724:2:1:NEW 20 Mar 2020) 
255 from the ECM-related biological samples (Table S8) (Åhrman et al. 2018; Naba et al. 2017).

256 There are a total of 1510 putative ECM proteins (1494 genes) in ECMPrideDB, and the official

257 gene symbols were used for comparison with other datasets. Overall, most ECM components in

258 Human Matrisome are included in ECMPrideDB ( 69.62\%, Fig. 3A). Specifically,

259 ECMPrideDB covers $\sim 92.33 \%$ of the Core matrisome and $\sim 61.35 \%$ of the Matrisome-

260 associated components in Matrisome (Fig. 3B). Additionally, 779 more novel ECM components

261 are found in ECMPrideDB. For the 21 Core matrisome components uniquely included in

262 Matrisome, 15 of them were also predicted as potential ECMs by ECMPride but with relatively

263 low confidence (probability $<0.7$ ). None of them were annotated with extracellular matrix terms

264 in GO. A similar situation also prevailed in the 291 Matrisome-associated components uniquely

265 included in Matrisome, about half of them $(153 / 291)$ were predicted as potential ECMs by

266 ECMPride with low confidence (probability $<0.7)$, and a vast majority of them $(275 / 291)$ were

267 annotated without extracellular matrix annotations in GO, indicating that there might be

268 insufficient evidence to support these proteins to be real ECMs and more biological or structural

269 information need to be explored.

270

271 For both proteomic experimental datasets, most of the identified proteins that overlap with

272 Matrisome are also contained in ECMPrideDB, and considerable numbers of novel ECMs (96

273 and 127, respectively, Fig. 3C and Fig. 3D) are found in ECMPrideDB.

274

275 Validation of novel ECM components

276 To further validate the putative ECM proteins predicted by ECMPride, several analyses were

277 implemented. Among the 779 putative ECMs uniquely identified in ECMPrideDB, 283 of them

278 contain at least one of the protein domains proposed by Naba et al. as the specific features for

279 Core Matrisome (Naba et al. 2016) (Table S7). It is due to the update of the underlying domain

280 annotation that these newly predicted ECMs emerge. To an extent, it also proves the reliability of

281 putative ECMs predicted by ECMPride. The presentation of experimental interactions with

282 known ECM proteins could be supportive evidence for the new putative ECMs. Thus, the

283 protein-protein interactions of the 779 putative ECMs with all ECMs in Matrisome are retrieved

284 both from MatrixDB (Clerc et al. 2018) and STRING (Szklarczyk et al. 2018) database. It is

285 found that 619 of 779 putative ECMs can interact with at least one known ECM in Matrisome.

286 Finally, the detailed interactions, as well as the hyperlink of the Entrez gene summary, are

287 provided with each putative ECM in ECMPrideDB in Table S7.

288

289 Further, we confirmed the expression of several potential novel ECM components in the top list

290 of the ECMPrideDB by immunohistochemistry and immunofluorescence experiments, including

291 stabilin family members STAB1 and STAB2, and the jagged canonical notch ligands JAG1 and

292 JAG2 (Details refer to Article S1). Our results indicate that all four molecules are expressed in

293 the extracellular space of epidermis and dermis (Fig. S2). Interestingly, STAB1, STAB2, and

294 JAG1 are specifically located in the basement membrane of skin tissue, which is the epidermal 
295

296

297

298

299

300

301

302

303

304

305

306

307

308

309

310

311

312

313

314

315

316

317

318

319

320

321

322

323

324

325

326

327

328

329

330

331

332

333

334

stem cell niches (Fig. S2A-C). And JAG2 is specifically located in the spinous, granular, and stratum corneum layers of the epidermis (Fig. S2D). Moreover, three new predicted ECM components (DLL4, LRP1, and FCGBP) are found expressed in the extracellular space of normal human liver and skin tissues, as well as RH-30 cell lines (Fig. S3). Although the current immunohistochemical and immunofluorescence experiments are not sufficient to verify that these proteins are ECM proteins, the results are nevertheless a useful preliminary validation, and more work remains to be done.

\section{Discussion}

More and more proteomics studies are applied for large-scale ECM protein identification, and the theoretical ECM database was used in these studies to identify ECM proteins and guide the biological analysis and experiments (Åhrman et al. 2018; Gopal et al. 2017; Lennon et al. 2014; Mayorca-Guiliani et al. 2017). Thus, the development of ECMs prediction methods and the construct of comprehensive ECM reference datasets are required and will benefit proteomicsbased ECM researches.

In this study, we proposed a flexible and scalable tool ECMPride for predicting extracellular matrix proteins by incorporating the advantages of experiment-based features and robust prediction models. There are three classes of features implemented in ECMPride to represent the characteristics of ECM proteins, including ECM protein-related structural domains (63 features), physicochemical properties (24 features), and position-specific scoring matrix (PSSM, 80 features). The physicochemical properties and PSSM have been used in many models and tools for the prediction of protein structure and function for multi-species (Chen \& Li 2013; Du \& Yu 2013; Hayat \& Khan 2012; Lundegaard et al. 2008). While for the features of domains, ECM proteins are highly conserved among different species, not only in the sequences of specific domains but also in the arrangements of those domains (Hynes 2009). Utilizing the conserved nature of domains across species, Naba et al. used the same list of domains to construct human and mouse ECM datasets, respectively (Naba et al. 2012). At present, we applied ECMPride to predict human ECM proteins, but we think ECMPride can be useful for ECM proteins prediction for other species.

Among all seven ECM prediction tools introduced in this study (ECMPP, EcmPred, PECM, IECMP, ECMP-HybKNN, BAMORF, and TargetECMP), four of them (ECMPP, EcmPred, PECM, and IECMP) were released with web-based applications. Unfortunately, none of these tools are currently available. Therefore, the maintenance and update of software tools are essential for public users. ECMPride is developed as an open-source and easy-to-use tool. To analysis the large datasets efficiently, we also designed a parallel version of ECMPride, which could perform the prediction of proteins with multi-threads mode. All the source codes of ECMPride with single-thread and multi-threads versions are publicly available from GitHub (https://github.com/Binghui-Liu/ECMPride.git). As the experimental validated ECM proteins 
335

336

337

338

339

340

341

342

343

344

345

346

347

348

349

350

351

352

353

354

355

356

357

358

359

360

361

362

363

364

365

366

367

368

369

370

371

372

373

374

and annotation database based features would keep updating, we will further improve the sensitivity and specificity of the prediction model and provide the continuously update service of the ECMPride tool. Based on ECMPride, we plan to develop a web-based database for reference ECM proteins for multi-species, which can provide a user-friendly web interface for browsing, searching, and downloading all putative ECM components, as well as the abundant biological annotations.

\section{Conclusions}

In this study, we developed ECMPride, a flexible and scalable tool for accurate and automatic prediction of ECM proteins. ECMPride can achieve excellent performance in predicting ECM proteins, with a relatively good balanced accuracy and sensitivity. By applying ECMPride to human protein sequences in SwissProt, a new dataset ECMPrideDB of all putative human ECM components was established. This dataset covers most known ECMs in Human Matrisome, and more potential ECM proteins are identified when using this dataset to annotate the experimental proteomics datasets. As ECMPride is developed based on the machine learning method, the robust of modeling makes it easy to deal with other species' proteins sequences in a similar way, i.e., mouse, rat, and so on. Also, with the accumulation of publicly available ECM proteomics datasets, more experimentally verified ECMs can be added into the standard dataset and further improve the model's prediction performance.

\section{Acknowledgements}

We thank Dr. Cheng Chang and Dr. Mansheng Li at the National Center for Protein Sciences (Beijing) for helpful discussion.

\section{References}

Åhrman E, Hallgren O, Malmström L, Hedström U, Malmström A, Bjermer L, Zhou X-H, Westergren-Thorsson G, and Malmström J. 2018. Quantitative proteomic characterization of the lung extracellular matrix in chronic obstructive pulmonary disease and idiopathic pulmonary fibrosis. Journal of Proteomics 189:23-33.

Ali F, and Hayat M. 2016. Machine learning approaches for discrimination of Extracellular Matrix proteins using hybrid feature space. Journal of Theoretical Biology 403:30-37.

Altschul SF, Madden TL, Schäffer AA, Zhang J, Zhang Z, Miller W, and Lipman DJ. 1997. Gapped BLAST and PSI-BLAST: a new generation of protein database search programs. Nucleic Acids Research 25:3389-3402.

Apic G, Huber W, and Teichmann SA. 2003. Multi-domain protein families and domain pairs: comparison with known structures and a random model of domain recombination. Journal of Structural and Functional Genomics 4:67-78.

Bateman JF, Boot-Handford RP, and Lamandé SR. 2009. Genetic diseases of connective tissues: cellular and extracellular effects of ECM mutations. Nature Reviews Genetics 10:173183. 
375 Bonnans C, Chou J, and Werb Z. 2014. Remodelling the extracellular matrix in development

376

377

378

379

380

381

382

383

384

385

386

387

388

389

390

391

392

393

394

395

396

397

398

399

400

401

402

403

404

405

406

407

408

409

410

411

412

413

and disease. Nature Reviews Molecular Cell Biology 15:786-801.

Bornberg-Bauer E, and Alba MM. 2013. Dynamics and adaptive benefits of modular protein evolution. Current Opinion in Structural Biology 23:459-466.

Breiman L. 2001. Random forests. Machine Learning 45:5-32.

Cain SA, McGovern A, Small E, Ward LJ, Baldock C, Shuttleworth A, and Kielty CM. 2009. Defining elastic fiber interactions by molecular fishing: an affinity purification and mass spectrometry approach. Molecular \& Cellular Proteomics 8:2715-2732.

Chen Y-K, and Li K-B. 2013. Predicting membrane protein types by incorporating protein topology, domains, signal peptides, and physicochemical properties into the general form of Chou's pseudo amino acid composition. Journal of Theoretical Biology 318:1-12.

Chou KC. 2001. Prediction of protein cellular attributes using pseudo - amino acid composition. Proteins: Structure, Function, and Bioinformatics 43:246-255.

Chou KC, and Shen HB. 2007. Large - scale plant protein subcellular location prediction. Journal of Cellular Biochemistry 100:665-678.

Clerc O, Deniaud M, Vallet SD, Naba A, Rivet A, Perez S, Thierry-Mieg N, and RicardBlum S. 2018. MatrixDB: integration of new data with a focus on glycosaminoglycan interactions. Nucleic Acids Research 47:D376-D381.

Consortium GO. 2016. Expansion of the Gene Ontology knowledgebase and resources. Nucleic Acids Research 45:D331-D338.

Consortium U. 2017. UniProt: the universal protein knowledgebase. Nucleic Acids Research 45:D158-D169.

Cromar GL, Xiong X, Chautard E, Ricard - Blum S, and Parkinson J. 2012. Toward a systems level view of the ECM and related proteins: a framework for the systematic definition and analysis of biological systems. Proteins: Structure, Function, and Bioinformatics 80:15221544.

Ding S, Yan S, Qi S, Li Y, and Yao Y. 2014. A protein structural classes prediction method based on PSI-BLAST profile. Journal of Theoretical Biology 353:19-23.

Du P, and Yu Y. 2013. SubMito-PSPCP: predicting protein submitochondrial locations by hybridizing positional specific physicochemical properties with pseudoamino acid compositions. BioMed Research International 2013:263829.

Gopal S, Veracini L, Grall D, Butori C, Schaub S, Audebert S, Camoin L, Baudelet E, Radwanska A, and Beghelli-de la Forest Divonne S. 2017. Fibronectin-guided migration of carcinoma collectives. Nature Communications 8:14105.

Guan L, Zhang S, and Xu H. 2017. BAMORF: A novel computational method for predicting the extracellular matrix proteins. IEEE Access 5:18498-18505.

Hayat M, and Khan A. 2012. MemHyb: predicting membrane protein types by hybridizing SAAC and PSSM. Journal of Theoretical Biology 292:93-102.

Hynes RO. 2009. The extracellular matrix: not just pretty fibrils. Science 326:1216-1219.

Peer) reviewing PDF | (2019:12:43724:2:1:NEW 20 Mar 2020) 
414 Jung J, Ryu T, Hwang Y, Lee E, and Lee D. 2010. Prediction of extracellular matrix proteins 415 based on distinctive sequence and domain characteristics. Journal of Computational Biology 416 17:97-105.

417 Kabir M, Ahmad S, Iqbal M, Swati ZNK, Liu Z, and Yu D-J. 2018. Improving prediction of 418 extracellular matrix proteins using evolutionary information via a grey system model and 419 asymmetric under-sampling technique. Chemometrics and Intelligent Laboratory Systems 420 174:22-32.

421 Kandaswamy KK, Pugalenthi G, Kalies K-U, Hartmann E, and Martinetz T. 2013. 422 EcmPred: Prediction of extracellular matrix proteins based on random forest with maximum 423 relevance minimum redundancy feature selection. Journal of Theoretical Biology 317:377-383. 424 Kawashima S, Pokarowski P, Pokarowska M, Kolinski A, Katayama T, and Kanehisa M. 425 2007. AAindex: amino acid index database, progress report 2008. Nucleic Acids Research 426 36:D202-D205.

427 Keerthikumar S, Chisanga D, Ariyaratne D, Al Saffar H, Anand S, Zhao K, Samuel M, 428 Pathan M, Jois M, and Chilamkurti N. 2016. ExoCarta: a web-based compendium of 429 exosomal cargo. Journal of Molecular Biology 428:688-692.

430 Lennon R, Byron A, Humphries JD, Randles MJ, Carisey A, Murphy S, Knight D, 431 Brenchley PE, Zent R, and Humphries MJ. 2014. Global analysis reveals the complexity of 432 the human glomerular extracellular matrix. Journal of the American Society of Nephrology 433 25:939-951.

434 Liaw A, and Wiener M. 2002. Classification and regression by randomForest. $R$ News 2:18-22. 435 Lin W-Z, Fang J-A, Xiao X, and Chou K-C. 2013. iLoc-Animal: a multi-label learning 436 classifier for predicting subcellular localization of animal proteins. Molecular BioSystems 9:634437644.

438 Liu N, Matsumura H, Kato T, Ichinose S, Takada A, Namiki T, Asakawa K, Morinaga H, 439 Mohri Y, and De Arcangelis A. 2019. Stem cell competition orchestrates skin homeostasis and 440 ageing. Nature 568(7752):344-350

441 Lundegaard C, Lamberth K, Harndahl M, Buus S, Lund O, and Nielsen M. 2008.

442 NetMHC-3.0: accurate web accessible predictions of human, mouse and monkey MHC class I 443 affinities for peptides of length 8-11. Nucleic Acids Research 36:W509-W512.

444 Matsuda S, Vert JP, Saigo H, Ueda N, Toh H, and Akutsu T. 2005. A novel representation of 445 protein sequences for prediction of subcellular location using support vector machines. Protein 446 Science 14:2804-2813.

447 Mayorca-Guiliani AE, Madsen CD, Cox TR, Horton ER, Venning FA, and Erler JT. 2017. 448 ISDoT: in situ decellularization of tissues for high-resolution imaging and proteomic analysis of 449 native extracellular matrix. Nature Medicine 23(7):890-898.

450 Mitchell AL, Attwood TK, Babbitt PC, Blum M, Bork P, Bridge A, Brown SD, Chang H-Y, 451 El-Gebali S, and Fraser MI. 2018. InterPro in 2019: improving coverage, classification and 452 access to protein sequence annotations. Nucleic Acids Research 47:D351-D360. 
453 Naba A, Clauser KR, Ding H, Whittaker CA, Carr SA, and Hynes RO. 2016. The

454 extracellular matrix: Tools and insights for the "omics" era. Matrix Biology 49:10-24.

455 Naba A, Clauser KR, Hoersch S, Liu H, Carr SA, and Hynes RO. 2012. The matrisome: in 456 silico definition and in vivo characterization by proteomics of normal and tumor extracellular 457 matrices. Molecular \& Cellular Proteomics 11:M111. 014647.

458 Naba A, Pearce OM, Del Rosario A, Ma D, Ding H, Rajeeve V, Cutillas PR, Balkwill FR, 459 and Hynes RO. 2017. Characterization of the extracellular matrix of normal and diseased tissues 460 using proteomics. Journal of Proteome Research 16:3083-3091.

461 Peng H, Long F, and Ding C. 2005. Feature selection based on mutual information: criteria of 462 max-dependency, max-relevance, and min-redundancy. IEEE Transactions on Pattern Analysis 463 \& Machine Intelligence:1226-1238.

464 Szklarczyk D, Gable AL, Lyon D, Junge A, Wyder S, Huerta-Cepas J, Simonovic M, 465 Doncheva NT, Morris JH, and Bork P. 2018. STRING v11: protein-protein association 466 networks with increased coverage, supporting functional discovery in genome-wide experimental 467 datasets. Nucleic Acids Research 47:D607-D613.

468 Theocharis AD, Skandalis SS, Gialeli C, and Karamanos NK. 2016. Extracellular matrix 469 structure. Advanced Drug Delivery Reviews 97:4-27.

470 Thul PJ, Åkesson L, Wiking M, Mahdessian D, Geladaki A, Blal HA, Alm T, Asplund A, 471 Björk L, and Breckels LM. 2017. A subcellular map of the human proteome. Science 472 356(6340):eaal3321.

473 Tokhmafshan F, Brophy PD, Gbadegesin RA, and Gupta IR. 2017. Vesicoureteral reflux and 474 the extracellular matrix connection. Pediatric Nephrology 32:565-576.

475 Walker C, Mojares E, and del Río Hernández A. 2018. Role of extracellular matrix in 476 development and cancer progression. International Journal of Molecular Sciences 19:3028.

477 Yang R, Zhang C, Gao R, and Zhang L. 2015. An ensemble method with hybrid features to 478 identify extracellular matrix proteins. PloS One 10:e0117804.

479 Zhang J, Sun P, Zhao X, and Ma Z. 2014. PECM: Prediction of extracellular matrix proteins 480 using the concept of Chou's pseudo amino acid composition. Journal of Theoretical Biology 481 363:412-418.

482 Zuo Y-C, Peng Y, Liu L, Chen W, Yang L, and Fan G-L. 2014. Predicting peroxidase 483 subcellular location by hybridizing different descriptors of Chou'pseudo amino acid patterns. 484 Analytical Biochemistry 458:14-19. 485 
Figure 1

Flowchart of the ECMPride pipeline.
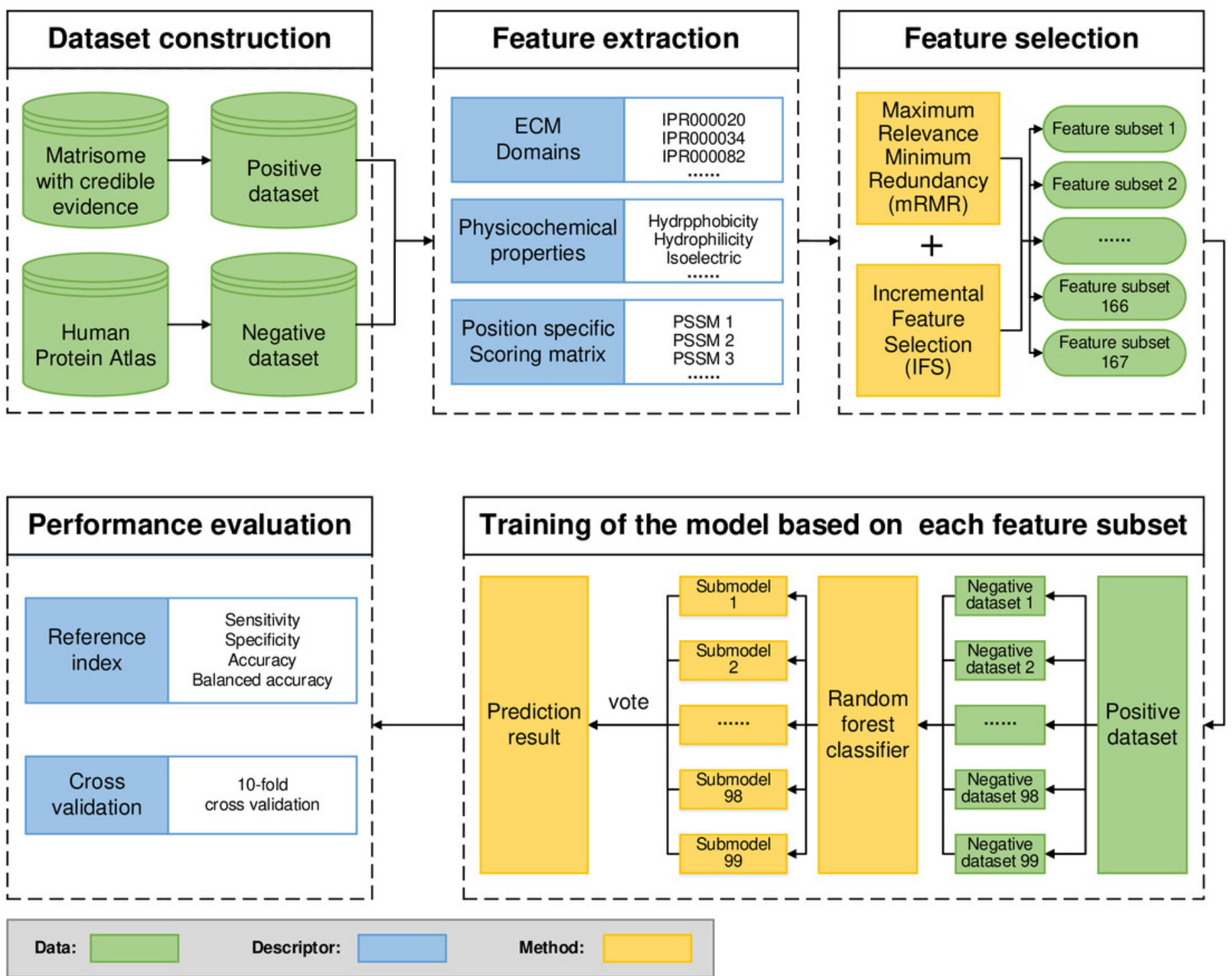
Figure 2

The feature selection curve of balanced accuracy for different feature subsets.

The 167 feature subsets were obtained by adding features one by one in order of importance from high to low. On the basis of each feature subset, the model was established with 10-fold cross-validation. The curve represents the relationship between the feature subset and its corresponding model's balanced accuracy.

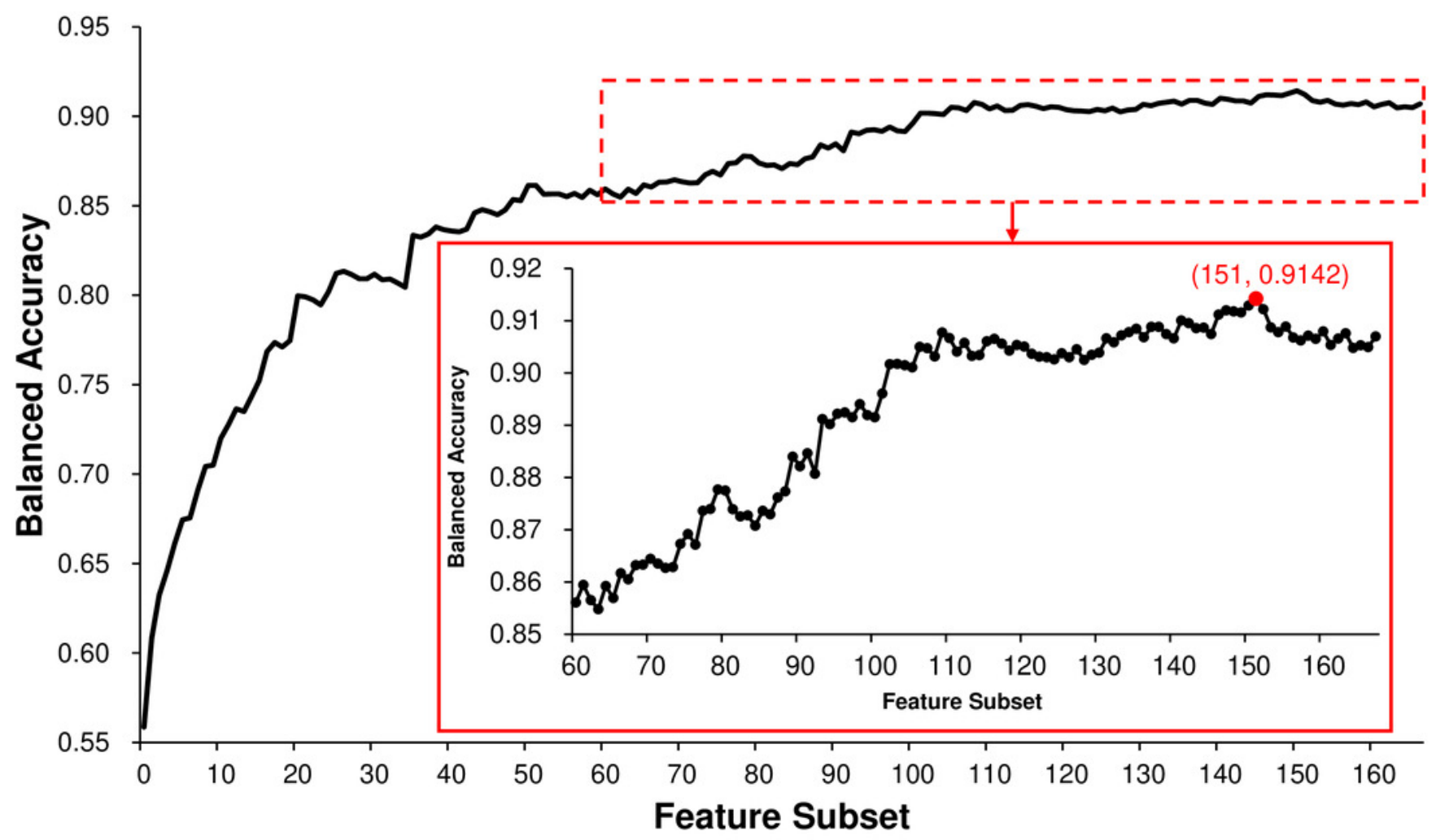


Figure 3

Comparison of the new ECM proteins with Human Matrisome and other experimental datasets.

The black, blue, and orange circles represent ECMPrideDB, Human Matrisome (dark blue for core matrisome and light blue for Matrisome-associated), and two proteomics experimental datasets, respectively. (A) Overlap of ECMPrideDB and Matrisome. (B) Overlap of ECMPrideDB, core Matrisome, and Matrisome-associated. (C) Overlap of ECMPrideDB, Matrisome, and ECM proteomics dataset of Naba et al. (D) Overlap of ECMPrideDB, Matrisome, and ECM proteomics dataset of Åhrman et al.

A

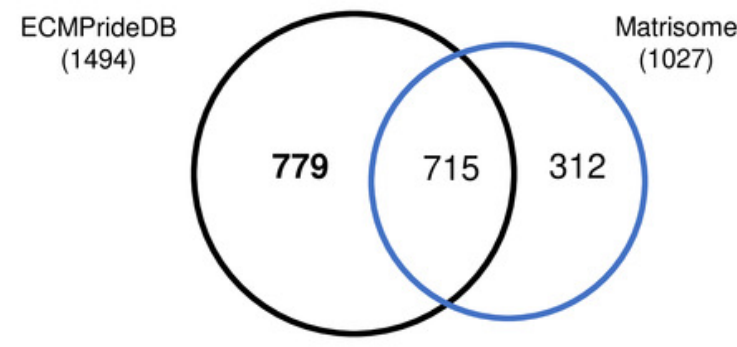

C

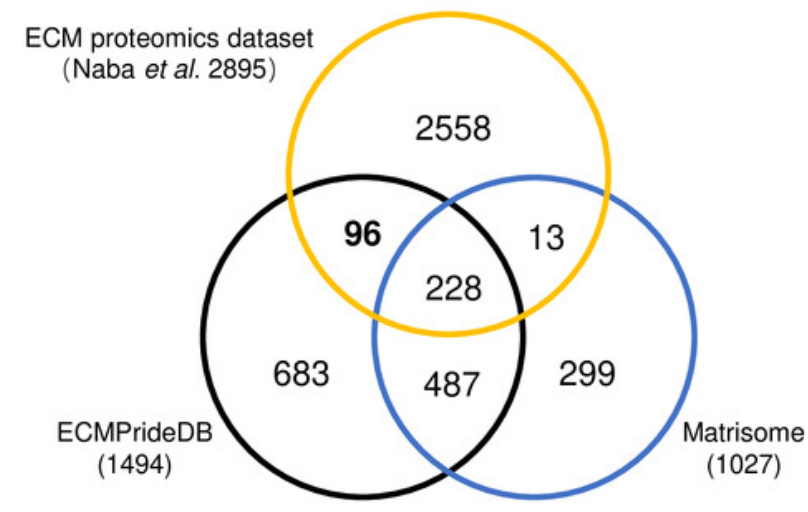

B

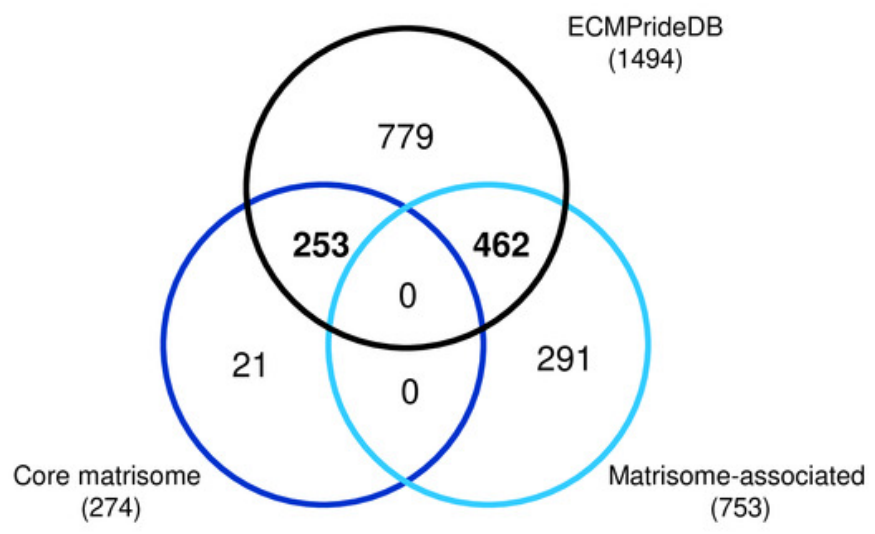

D

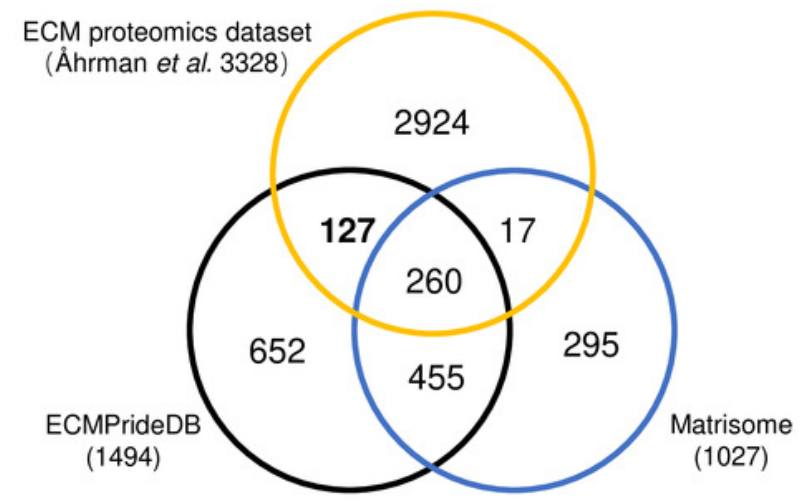




\section{Table $\mathbf{1}$ (on next page)}

Performance comparison of models with different methods and datasets. 
1 Table 1:

2 Performance comparison of models with different methods and datasets.

\begin{tabular}{llllll}
\hline Method & Dataset & Sensitivity & Specificity & Accuracy & Balanced accuracy \\
\hline \multirow{2}{*}{ ECMPride } & D1 & 0.8925 & 0.9360 & 0.9340 & 0.9142 \\
& D2 & 0.8783 & 0.8623 & 0.8638 & 0.8703 \\
EcmPred & D1 & 0.8462 & 0.9158 & 0.9145 & 0.8810 \\
& D2 & 0.6500 & 0.7700 & 0.8300 & 0.7100 \\
\hline
\end{tabular}

3 D1: Training dataset constructed in ECMPride's model.

4 D2: Training dataset constructed in EcmPred's model. 